\title{
PASSIVE AND ACTIVE TASKS OF SOUND RECOGNITION AND LOCALIZATION: A HUMAN fMRI STUDY
}

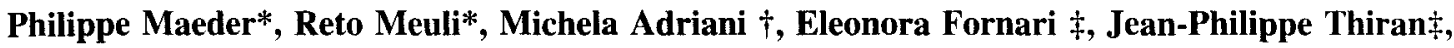 \\ Antoine Pittet§, Stephanie Clarke $\nmid$
}

* Department of Diagnostic and Interventional Radiology, University Hospital, Lausanne, Switzerland

$\dagger$ Department of Neuropsychology, University Hospital, lausanne, Switzerland

$\ddagger$ Signal Processing Laboratory, Swiss Federal Institute of Technology, lausanne, Switzerland

$\S$ Engineer School of Geneva, Geneva, Switzerland

\section{Purpose:}

In a separate study we have demonstrated different pathways for auditory spatial and recognition tasks (1) We now study the effect of active versus passive auditory tasks.

\section{Methods:}

Brain activations associated with learning in tasks of sound localization in space and sound recognition were investigated in 8 human subjects, using fMRI. In the localization task the subject had to identify and compare the spatial positions of the stimuli. In the recognition task, the subject had to identify and classify the nature of the sounds presented. Both types of stimuli were presented during the same fMRI acquisition in a triple epoch related study (localization - recognition - rest). A long TR and careful adjustment of the timing of the stimuli presentation allowed to acquire fMRI data without interference with the gradient noise.(2) Eight right-handed volunteers between age 24 and 47 were studied The right-handedness was established by Oldfield questionnaire. The subject had no history of neurological illness, there were 3 female and 5 male. Auditory stimuli of a total duration of 5 seconds consisted in a background and a target. For stimuli with spatial content the background was made of 25 samples of whitenoise of 15 milliseconds duration each, with variable DIT simulating a variable location in the horizontal plane. Stimuli were presented approximately every 200 milliseconds. The target consisted of filtered white noise, lasting 500 milliseconds. The first target was presented 1.5 seconds after the beginning of the background; the second after 3 seconds. Both stimuli were lateralized on the same side. Each subject was first investigated as passive,without any instruction. In the second part of the study, the active subject was instructed to press a pneumatic device with the right hand when the two stimuli had a different spatial localization and when an animal sound was recognized. Images were mosaics of 16 slices covering the whole hemispheres in the bicomissural plane. Postprocessing was conducted separately on a Silicon Graphic Indigo 2 workstation with SPM 99 software (the welcome Department of cognitive neurology, Queen's Square, London). After coregistering and reslicing, images were normalized and smoothed. The results from 8 subjects were pooled.

Regions of the brain that were more activated during the active task than during the passive task were depicted separately in both localization and recognition tasks.

\section{Results:}

In both conditions, primary temporal auditory cortex was strongly activated bilaterally. In the passive task, regions that were morc activated in recognition than localization included temporal convexity(centered on middle temporal gyrus) and posterior prefrontal cortex on both sides. Regions that were more activated in localization than recognition included inferior part of inferior parietal lobule bilateraly. In the active tasks additional regions were recruited selectively: for recognition, the precuneus bilateraly and the inferior temporal gyrus on the left side, for localization upper part of inferior parietal lobule and intraparietal sulcus(more on the right than on the left side) and posterior part of middle frontal and inferior frontal gyrus.

\section{Conclusion:}

These findings demonstrate that larger cortical networks are involved in active than passive tasks. These networks remain separate for sound recognition and localization.

\section{References:}

1) MaederP., Meuli R., Adriani M. et al. Distinct pathways involved in sound recognition and localization: a human fMRI study. submitted to HBM 2000

2) Meuli R., Maeder P., Adriani M. et al, Optimisation of stimuli and acquisition technique for tMRl of the auditory system submitted to HBM 2000 